\title{
A. K. Frolkova, "Razdelenie azeotropnykh smesei: Fiziko-khimicheskie osnovy i tekhnologicheskie priemy" (Separation of Azeotropic Mixtures: Physicochemical Principles and Techniques), Moscow: Vlados, 2010
}

\author{
DOI: $10.1134 / \mathrm{S} 0040579511050320$
}

One of the core problems of the bulk chemical industry is to analyze and synthesize flowsheets and functional complexes for isolation of the target products. Azeotropic systems are very frequent in industry, so separation of mixtures containing azeotropes formed by various numbers of components attracts attention of many researchers both in Russia and abroad. In her monograph, A.K. Frolkova systematizes, using thermodynamic and topological analysis methods, azeotropic separation data obtained to date by the scientific school of the Moscow State Academy of Fine Chemical Technology and by other researchers.

The monograph consists of four chapters, an extensive bibliography, and two appendices. One of the latter presents a comprehensive survey of separation techniques for physicochemically complex liquid mixtures, and the other highlights the relevant works of Russian scientists. Either one has its own list of references.

The monograph considers azeotropy in binary and multicomponent mixtures, the evolution of the composition of azeotropic mixtures under variations of external conditions, the evolution of azeotropic mixtures in the presence of other substances, and the separation of azeotropic mixtures using functional complexes. Each chapter demonstrates the correlation between the structure of the phase equilibrium diagram and the set of possible functional complexes consisting of a number of distillation columns coupled by recycle streams. The separation thermodynamics, principles, and techniques are considered within a unified systems approach to separation of complex mixtures, including azeotropic, heteroazeotropic, extractive, and reextractive distillation and separation involving a medium-boiling agent.

This monograph is the first to systematize data concerning biazeotropy, the phenomenon in which a binary mixture has two binary azeotropes or a threecomponent mixture has two ternary azeotropes. A number of such mixtures have been encountered in industry.

The monograph covers a wide variety of problems, including the physicochemical features of complicated binary and multicomponent mixtures, possible methods for their separation, and use of concentration field redistribution between distillation regions. The problems are addressed at the up-to-date theoretical level. The author's recommendations as to selection of separation systems and techniques are of great practical utility. The book is intended for researchers and engineers engaged in the chemical, oil refining, petrochemical, and related industries. It might also be helpful for teachers, students, and postgraduates of chemical engineering universities. 